Research Article

\title{
Chaotic Oscillations in a Third Order PLL in the Face of Two Co-Channel Signals and Its Control
}

\author{
B. C. Sarkar ${ }^{* 1}$ and S. Chakraborty ${ }^{2}$ \\ ${ }^{I}$ Physics Department, Burdwan University, Burdwan-713104, West Bengal, India. \\ ${ }^{2}$ Physics Department, Bidhan Chandra College, Asansol- 713304, West Bengal, India.
}

Received 22 September 2014; Revised 24 October 2014; Accepted 5 November 2014

\begin{abstract}
A third order phase locked loop incorporating a resonant type second order filter is a conditionally stable system and shows complicated dynamics including chaotic oscillations for a range of loop parameters. In the face of two co-channel signals, an otherwise stable loop may be thrown into a chaotic state, depending on the relative strength and mutual frequency offset of the input signals. We have predicted the parameter zone for chaotic state of the loop through numerical studies and verified the prediction by hardware experiment. Then we modify the loop structure to incorporate an additional control signal which stabilizes the loop dynamics and removes the chaotic oscillations. The improved response of the loop is established numerically and experimentally.
\end{abstract}

Keywords: Third order Phase Locked Loop, chaotic dynamics, co-channel interference, proportional-Integrating-derivative control, Lyapunov exponent.

\section{Introduction}

The application of phase locked loops (PLLs) in several electronic systems is well documented in the literature $[1,2]$. Particularly, they are integral parts of communication systems for carrier regeneration, angle demodulation or clock and data recovery. Generally second order PLLs with first order loop filters are predominantly used in practical systems, but third order PLLs having second order filters also find applications in specific cases [2]. Besides in conventional analog PLLs, the phase locking principle based systems are implemented using charge pump type filters or digital building blocks. The dynamics of these charges pump PLLs and digital PLLs in third order varieties has been extensively studied in the literature [3-6].

In real life communication systems the presence of cochannel interference signals is highly probable and it modifies the amplitude and phase statistics of the received signal [7]. The phase error dynamics of conventional PLLs gets perturbed because of the presence of the interference signal and modified loops incorporating a derivative type control signal in the loop structure is proposed to combat the effects of interference in digital PLLs [8]. Moreover chaotic oscillations have been observed in autonomous third order or higher order analog PLLs [9- 11]. Even second order PLLs

* E-mail address: bcsarkar_phy@yahoo.co.in ISSN: 1791-2377 (C) 2015 Kavala Institute of Technology. All rights reserved. subjected to more than one input signals [12] have been found to become chaotic. Also the second order PLLs employed to demodulate frequency/ phase modulated input signals [13] show chaotic response under certain conditions. When the propagation delay of signals in a PLL is not negligible, chaotic oscillations of the control signal may be observed.

In this work we study the dynamics of a third order PLL comprising of a Sallen-Key type second order resonant loop filter. This system is potentially chaotic as has been reported in the literature $[9,11]$. However, by a suitable choice of the loop design parameters it can be operated in a stable zone of operation for a single input signal. But when driven by two co-channel signals the dynamics may become chaotic and may cause problems for a system designer. In the context of ever increasing number of radio channel users, the possibility of having more than one signal at the input of a PLL receiver is very likely. In this paper we have obtained the conditions of chaotic oscillation for the loop driven by two signals in terms of their frequency difference and amplitude ratio. Then we propose a circuit modification to control the chaos. For this, the control signal applied to the loop voltage controlled oscillator (VCO) is modified to get both frequency and phase modulation of the loop VCO.

The paper is organized in the following way. The functional structure and the system equation of the third order PLL with resonant type filter is given in the next section. We have studied the dynamics of the loop by numerically finding the time evolution of the state variables 
of the PLL with two input signals. It shows the chaotic transition of the VCO control signal when the frequency offset and the amplitude of the second signal cross limiting values. We calculate the Lyapunov exponent (LE) spectrum of the loop state variables to examine the influence of the signal parameters on the transition to a chaotic state of the loop quantitatively. Then a modification of the circuit is proposed to control the chaotic oscillations is given in section 3. In the same section the dynamics of the modified system has been numerically studied and also compared with that of the conventional PLL. Experimental results are given in section 4 to establish the ability of chaos control of the modified circuit. In section 5 some concluding remarks on the study have been given.

\section{Dynamics of the Conventional PLL}

The functional block diagram of a third order PLL with two co-channel input signals is shown in Fig. 1. The figure incorporates the modification circuit proposed in this paper, however we discuss with that circuit in Section 3. First we consider one input signal written as, $A \sin \left(\omega_{i} t+\theta_{i}\right)$, where $A, \omega_{i}$ and $\theta_{i}$ represent the amplitude, the angular frequency and the constant phase of the signal. The output of the loop VCO is taken as, $2 \cos \left(\omega_{r} t+\theta_{r}\right)$, where $\omega_{r}$ is the free running angular frequency of the VCO and, in the closed loop condition $\theta_{r}$ is the time integrated version of the VCO control signal, multiplied by the VCO sensitivity $\left(k_{v}\right)$. The multiplier type phase detector detects the phase error between its inputs. In the absence of the second input signal, we write the phase difference between two inputs as $\varphi=\left[\left(\omega_{i}-\omega_{r}\right) t+\left(\theta_{i}-\theta_{r}\right)\right]$, or equal to $\left[\Omega t+\left(\theta_{i}-\theta_{r}\right)\right]$.

Here $\Omega$ is the frequency difference between the input signal and the VCO signal. The loop filter as shown in Fig. 1 , is a resonant type second order filter and in terms of the Heaviside operator $p(=d / d t)$, we write the time domain representation of the filter as,

$$
F(p)=\frac{g}{p^{2} T^{2}+(3-g) p T+1}
$$

Here the filter gain $g\left(=1+\left(R_{2} / R_{1}\right)\right)$ and the filter time constant $T(=R C)$ are used as loop design parameters. Using the definition of $\theta_{r}$, one can write the phase error equation of the PLL as:

$$
p \theta_{r}=A k_{v} F(p) \sin (\varphi)
$$

This can be reduced to the following form:

$$
\frac{d \varphi}{d t}=\Omega-A k_{v} F(p) \sin (\varphi)
$$

Now we consider the input to the PLL as a sum of two signals of respective amplitudes $A$ and $m A$ ( $m$ is a number less than one), angular frequencies $\omega_{i}$ and $\left(\omega_{i}+\Delta \omega\right)$ $\left(\Delta \omega<<\omega_{i}\right)$ and constant phase angle $\theta_{i}$. Hence we can write the resultant input signal with a time varying amplitude and phase angle as $A \rho(t) \sin \left(\omega_{i} t+\theta_{i}+\psi(t)\right)$, where,

$$
\begin{aligned}
& \rho(t)=\sqrt{\left(1+m^{2}+2 m \cos (\Delta \omega t)\right)} \\
& \psi(t)=\arctan (m A \sin (\Delta \omega t) /(1+m A \cos (\Delta \omega t)))
\end{aligned}
$$

When two co-channel signals are present at the input of the loop, the output of the phase detector is proportional to $\rho(t) \sin (\varphi+\psi)$. Using the phase domain model of the loop shown in Fig 1, the phase governing equation for the loop, in the operator notation would be,

$$
\frac{d \mathrm{n}}{d t}=\Omega-A k_{v} F(p) \rho(t) \sin (\varphi+\psi)
$$

Note that, here the modification circuit has not been taken into account to formulate the equations. Knowing the form of $F(p)$ as given in (1) we get the system equation as:

$$
T^{2} \dddot{\varphi}+(3-g) T \ddot{\varphi}+\dot{\varphi}+A k_{v} g \rho(t) \sin (\varphi+\psi)=\Omega
$$

This is a third order differential equation in $\varphi$ with time dependent loop parameters and it indicates a complicated dynamics.

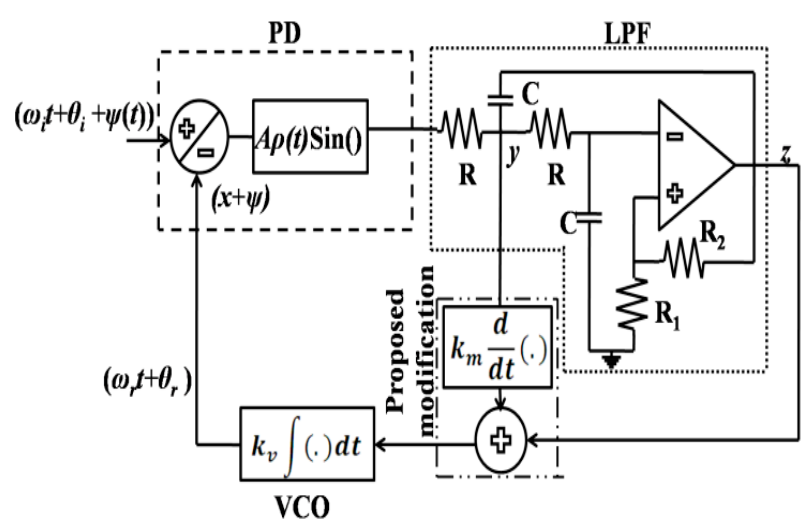

Fig. 1. Functional structure of the third order PLL with resonant second order LPF. The proposed circuit modification is shown in the dotted box. The state variables $x, y$ and $z$ are depicted in the figure.

To proceed further, we introduce three new state variables $x, y$ and $z$ for the loop. The loop phase error $\varphi$ is taken as a quantity reduced within $-\pi$ to $+\pi$ and it is chosen as the first state variable $x$ of the loop. The other two variables $y$ and $z$ are defined by following relations. One of the reasons of choosing $y$ and $z$ as state variables is that they could be directly measured at appropriate nodes of the circuit as shown in the block diagram of the loop. We take,

$$
\begin{aligned}
& y(t)=\left[\frac{(1+T p)}{p^{2} T^{2}+(3-g) p T+1}\right] \rho(t) \sin (\varphi(t)+\psi) \\
& z(t)=\left[\frac{g}{p^{2} T^{2}+(3-g) p T+1}\right] \rho(t) \sin (\varphi(t)+\psi)
\end{aligned}
$$

Using these state variables we decompose (5) into a set of state equations. These equations are written in terms of the normalized loop parameters $\Omega_{n}, k_{n}, \Omega_{i}$ and $\tau$ which are $\Omega T$, $A k_{v} T,(\Delta \omega) T$ and $(t / T)$ respectively. The set of state equations are as follows: 


$$
\begin{aligned}
& \frac{d x}{d \tau}=\Omega_{n}-k_{n} z \\
& \frac{d y}{d \tau}=\rho \sin (x+\psi)+(g-2) y-\left(\frac{g-1}{g}\right) z \\
& \frac{d z}{d \tau}=g y-z \\
& \frac{d \rho}{d \tau}=\Omega_{i} \sin (\psi) \\
& \frac{d \psi}{d \tau}=\Omega_{i}\left[\frac{m^{2}-1}{\rho^{2}}+\frac{\cos (\psi)}{\rho}\right]
\end{aligned}
$$

In the absence of the second signal at the input $(m=0)$, we put $\rho(t)$ and $\psi(t)$ as 1 and 0 respectively in (7a), (7b) and (7c) while other two equations need not be considered. For a given value of $\Omega_{n}$ and $k_{n}$, we examine the effect of variation of $g$ on the loop dynamics. The loop becomes self oscillatory when the value of $g$ becomes equal to a critical value [11]. For example, with $\Omega_{n}=0$ and $k_{n}=0.63$, one gets the condition of self oscillation in the PLL for a minimum limiting value of $g=1.84$. The frequency of self oscillation is a characteristic frequency, $\Omega_{C}$, of the third order PLL. An approximate estimate of this frequency can be obtained from the linearized loop equations at the condition of zero initial detuning. We assume a sinusoidal type oscillatory solution of the loop equation and apply the method of harmonic balance to obtain the frequency of self oscillation. This gives the value of $\Omega_{C}=\left[k_{n} g /(3-g)\right]^{1 / 2}$. Thus, with $g=1.705$ and $k_{n}=0.63$ we have the characteristic frequency $\Omega_{C}=0.9$.

In this work we keep the loop parameters in the stable zone of operation and consider the effect of a second signal having a frequency offset $\Omega_{i}$, with respect to that of the strong input signal. In this situation, the loop dynamics is described by the set of five equations given in (7). In presence of the second input signal, the VCO control signal would become time varying. For low values of $m$ the nature of the signal would be sinusoidal and its frequency is same as that of off-set frequency, $\Omega_{i}$. Increasing the value of $m$ for a fixed value of $\Omega_{i}$, comparable to $\Omega_{C}$, we note the amplitude of the oscillatory VCO control signal and plot the amplitude as a function of $m$. As shown in Fig. 2, with $g=1.705$ and $k_{n}=0.63$, when $m$ is 0.668 , the control signal becomes a combination of two oscillations. It is evident from two instantaneous amplitude values in time domain. For increasing values of $m$ the sequence of period doubling continues and ultimately the control signal of the VCO becomes chaotic in nature. We calculate different Lyapunov exponents (LEs) associated with the five state variables used in the state equations (7) of the system applying the algorithm suggested by Wolf et al [14]. The three largest LEs thus obtained are plotted in Fig. 3 as functions of $m$. The nature of the curves indicates the occurrence of period doubling bifurcation at specific values of $m$ and for $m>0.769$ we get one of the LEs positive. In this range of $m$, the chaotic oscillation of the VCO control signal is observed in Fig. 2. Thus the numerical studies of evaluating LEs and finding the time evolution of loop state variable give consistent results. We study the effects of variation of $\Omega_{i}$ keeping $m$ value fixed and it is observed that the loop dynamics becomes very much susceptible to the second signal when $\Omega_{i}$ is close to $\Omega_{C}$. The numerically observed results are summarized through bifurcation diagram in Fig. 4. The corresponding LE spectrum has been calculated as a function of $\Omega_{i}$ and three largest LEs are plotted in Fig. 5. One of the LEs become positive in between 0.69 $<\left|\Omega_{i}\right|<0.86$ indicating the chaotic nature of the system.

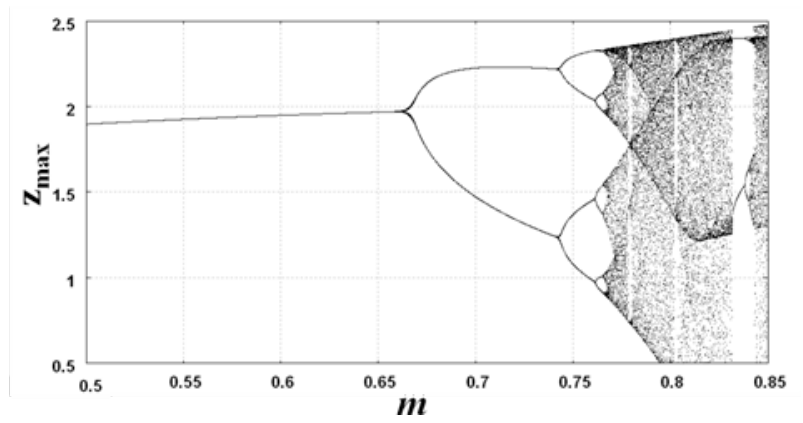

Fig. 2. Numerically obtained variation of the instantaneous maximum amplitude of the oscillatory control signal $z_{\max }$ as a function of $m$ for a CTOPLL. The values of other loop parameters are $\Omega_{n}=0, k_{n}=0.63$, $g=1.705$ and $\Omega_{i}=0.84$.

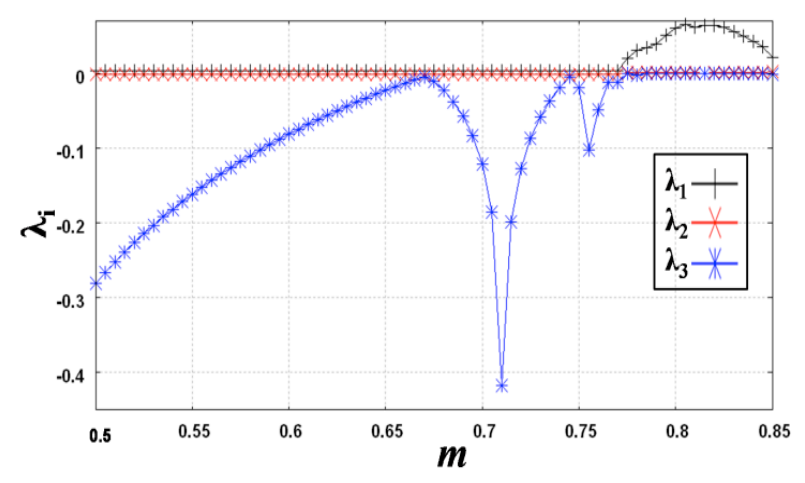

Fig. 3. Variation of three largest Lyapunov exponents of the CTOPLL with $m$ as the control parameter obtained applying Wolf's algorithm in state equations (3). The values of other loop parameters are $\Omega_{n}=0$, $k_{n}=0.63, g=1.705$ and $\Omega_{i}=0.84$.

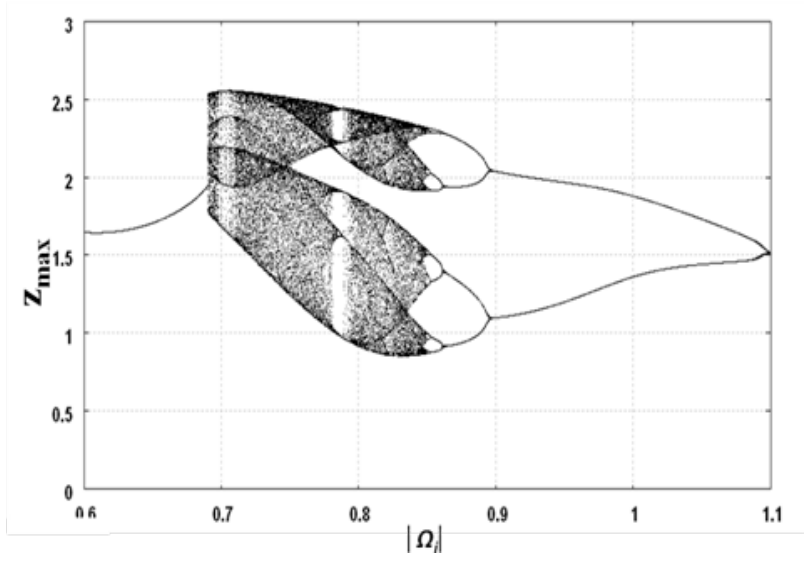

Fig. 4. Numerically obtained variation of the instantaneous maximum amplitude of the oscillatory control signal $z_{\max }$ as a function of $\Omega_{i}$ for a CTOPLL. The values of other loop parameters are $\Omega_{n}=0, k_{n}=0.63$, $g=1.705$ and $m=0.77$. 


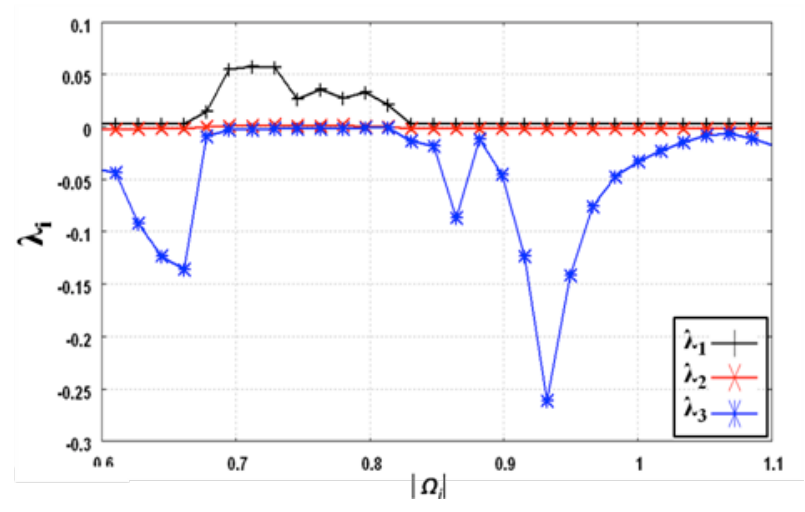

Fig. 5. Variation of three largest Lyapunov exponents of the CTOPLL with $\Omega_{i}$ as the control parameter obtained applying Wolf's algorithm in state equations (7). The values of other loop parameters are $\Omega_{n}=0$, $k_{n}=0.63, g=1.705$ and $m=0.77$.

\section{Dynamics of the Modified Loop}

We propose to include an additional sub-circuit in the PLL structure to control the adverse effect of the second signal in the loop dynamics and improve the stability of the loop. When the modification circuit is included, as shown in Fig. 1 , the VCO control signal is changed to $z_{c}(t)$ which is a sum of $z(t)$ and $k_{m}$ times the time derivative of $y(t)$. Thus, remembering the definitions of $y(t)$ and $z(t)$ given in (6), we note that the modified VCO control signal has terms proportional to $\sin (x+\psi)$, as well as first order and second order time derivatives of $\sin (x+\psi)$. Since the estimated phase by the loop VCO is time integrated version of applied control signal, it has components directly proportional to, integrated version of and time derivative of $\sin (x+\psi)$. Thus the effective loop filter becomes a proportional-integratingderivative type. It can be anticipated that the modified loop would have better stability performance.

With the modification, a new set of state equations would be obtained. However in the new set, only the time derivative of $x$ variable would be different from (7a) and it is given by the following (8). The time derivatives of $y, z, \rho$ and $\psi$ would remain same as given in (7b) to (7e) respectively. The changed equation of the time derivative of $x$ in the modified system is,

$$
\begin{aligned}
\frac{d x}{d \tau}= & \Omega_{n}-k_{n} k_{m} \rho \sin (x+\psi)-k_{n} k_{m}(g-2) y- \\
& -k_{n}\left\{1-k_{m}\left(\frac{g-1}{g}\right)\right\} z
\end{aligned}
$$

We have studied the dynamics of the proposed modified loop for different values of the parameter $k_{m}$ and examine the relative responses of the CTOPLL $\left(k_{m}=0\right)$ and modified PLL $\left(k_{m} \neq 0\right)$ with same set of values of $m$ and $\Omega_{i}$. The obtained responses are depicted through phase plane plots of state variables $y$ and $z$ as given in Fig. 6. It shows that the CTOPLL has a chaotic attractor but the modified PLL has a limit cycle type phase plane plot. In presence of the second co-channel signal at the input, the control signal is periodically modulated and we get the limit cycle type phase plane plot even in the stable mode of operation. Also we examine the effect of additional control signal by finding the LE spectrum of the system in the chaotic region of operation with $k_{m}$ as a control variable. The favourable effect of additional control signal in taming the chaos is evident from Fig.7. It indicates that taking a suitable range of value for $k_{m}$, the maximum LE (MLE) of the system could be made nonpositive value. This means that the dynamics of the loop could be brought back to non-chaotic state by choosing suitable $k_{m}$. The improved response of the modified third order PLL is due to the inclusion of effective proportionalintegrating-and-derivative control type filter in the loop.

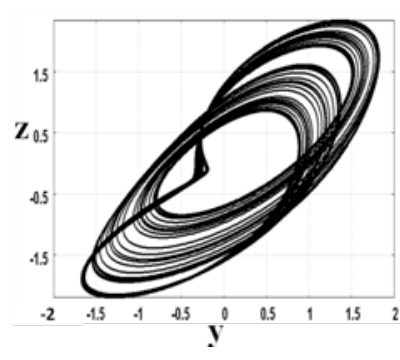

(a)

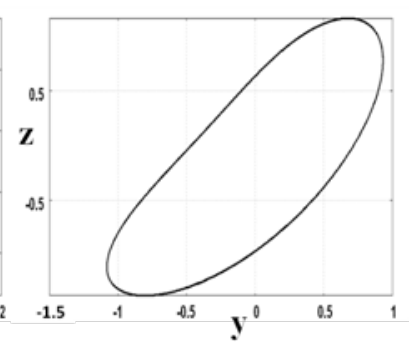

(b)
Fig. 6. Numerical simulation results showing the phase plane plots of conventional and modified PLL. The parameters taken are $\Omega_{n}=0$, $k_{n}=0.63, g=1.705, m=0.78$ and $\Omega_{i}=0.84$. Here, (a) represents the chaotic state in $\operatorname{CTOPLL}\left(k_{m}=0\right)$ and (b) represents the periodic state of DDCPLL $\left(k_{m}=1\right)$ with the same second signal.

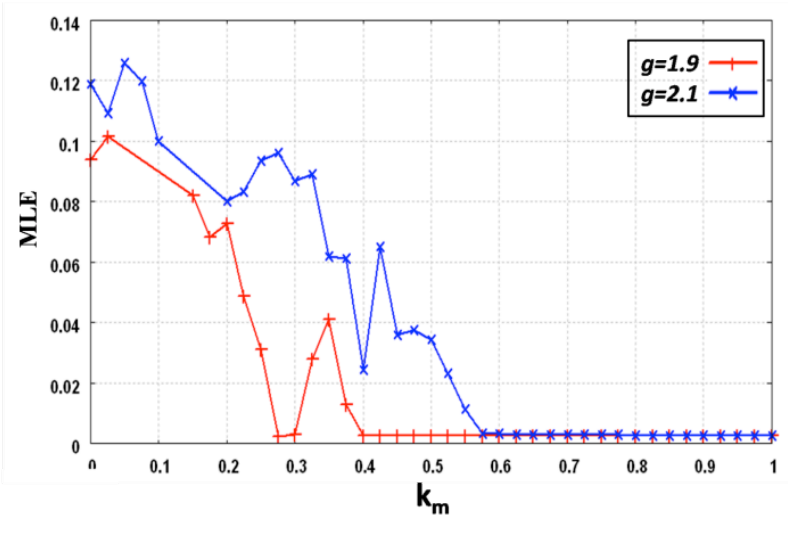

Fig.7. Variation of MLE of the modified PLL with $k_{m}$ as the control parameter obtained applying Wolf's algorithm. The values of other loop parameters are $\Omega_{n}=0, k_{n}=0.63, \quad g=1.705, \quad m=0.78$ and $\Omega_{i}=0.84$.

\section{Experimental Results}

A prototype hardware circuit of CTO-PLL has been designed using off-the-shelf ICs like 8038 (voltage controlled oscillator), AD532 (multiplier for phase detector), $\mu \mathrm{A} 0741$ (op-amp for active filter) etc for experimental studies. We design a resonant loop filter whose time constant is adjusted at $18 \mu \mathrm{s}$ and the gain is adjustable with the help of the resistor $R_{1}$ and $R_{2}$ (Fig. 1). The voltage sensitivity of the VCO is experimentally measured to be $29.8 \mathrm{kHz} / \mathrm{V}$. In the experiment we take the amplitude and frequency of the desired input signal as $1.1 \mathrm{~V}$ and $125 \mathrm{kHz}$ while those for the VCO output signal as $3.5 \mathrm{~V}$ and $125 \mathrm{kHz}$ respectively. Thus the frequency detuning between the desired signal and the VCO signal is taken as zero. The adjusted gain value of the CTO-PLL is 1.705 which is in 
stable zone of operation. Now the frequency of second signal applied by adding with the strong input signal is kept fixed at $117 \mathrm{kHz}$ while its amplitude is gradually increased. Thus the frequency difference between two input signals is 8 $\mathrm{kHz}$ which is nearly equal to the characteristic frequency of the loop filter. The experimental variation of $y$ and $z$ variables as observed is shown in Fig. 8. Here period-1, period-2, period- 4 and chaotic type phase-plane diagrams are obtained as we increase the amplitude of second signal. The results are consistent with the numerical prediction. Next we fix the strength of second signal at 0.86 volts (i.e. $m=0.78$ ) and vary its frequency. Experimental observations show that the conventional PLL becomes chaotic when the frequency difference between the two input signals is near the characteristic frequency of the resonant filter. The experimental results are shown in Fig. 9. The chaotic oscillation is obtained for the normalized frequency off-set in the range $-0.96<\Omega_{i}<-0.73$. Thus the experimental results are in qualitative agreement with the numerical prediction.

In the second part of the experiment, we have studied the dynamics of the proposed modified loop for different values of the parameter $k_{m}$. We examine the response of the CTOPLL $\left(k_{m}=0\right)$ and that of modified PLL $\left(k_{m}=1\right)$ with same set of values of $m$ and $\Omega_{i}$. In this case the design parameters are so chosen that the CTOPLL will give chaotic dynamics. Then under same condition we observe the dynamics of modified PLL with $k_{m}$ as a control parameter. The effect of $k_{m}$ in controlling chaos is observed. The experimentally observed results are given in Fig. 10 and it is consistent with the numerical predictions.

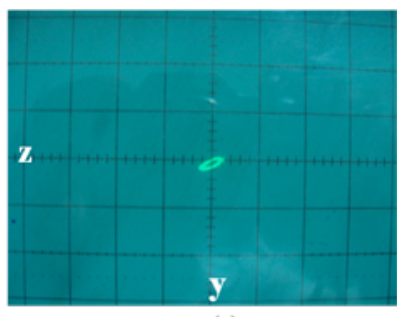

(a)

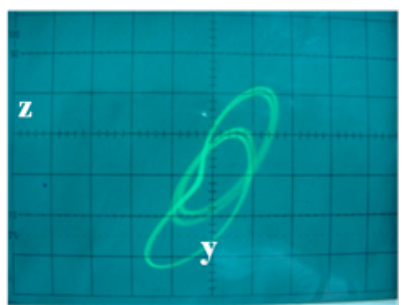

(c)

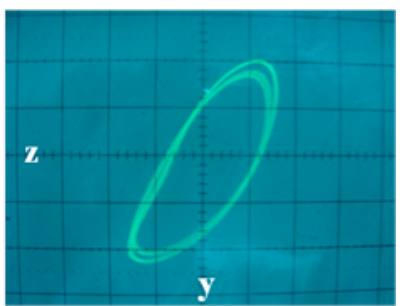

(b)

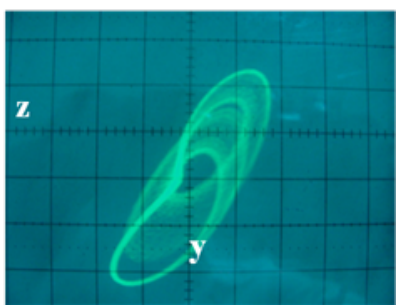

(d)
Fig. 8. Experimental results showing the period doubling route to chaos of the CTOPLL in presence of two input signals with system parameters: $\Omega_{n}=0, k_{n}=0.63, g=1.7$ and $\Omega_{i}=0.9$ by varying $m$; (a) $m=0.2$ (period-1 limit cycle), (b) $m=0.668$ (period-2 limit cycle), (c) $m=0.745$ (period-4 limit cycle), (d) $m=0.772$ (chaotic state).

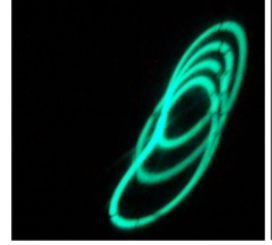

(a)

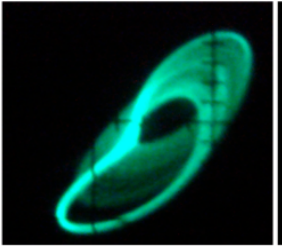

(b)

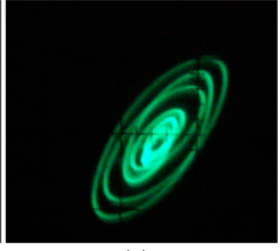

(c)
Fig. 9. Experimental results showing the system dynamics in presence of two input signals for the CTOPLL with parameters: $\Omega_{n}=0$, $k_{n}=0.63, g=1.7, m=0.78$ and varying the frequency difference $\left(\Omega_{i}\right)$ between two input signals; (a) $\Omega_{i}=0.98$, (b) $\Omega_{i}=0.9$, (c) $\Omega_{i}=0.2$.

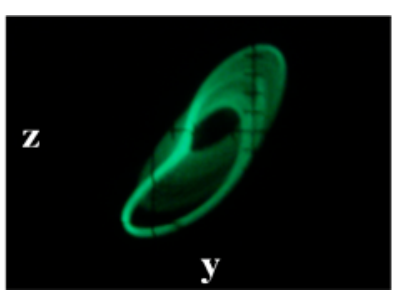

(a)

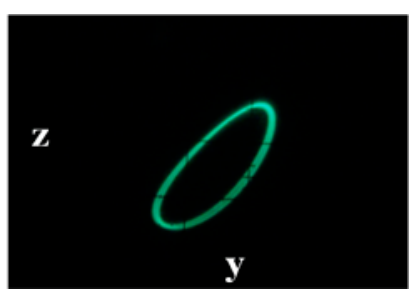

(b)
Fig. 10. Experimental results showing the phase plane plots of conventional and modified PLL. The normalized experimental parameter values are $\Omega_{n}=0, \quad k_{n}=0.63, \quad g=1.7, \quad m=0.78$ and $\Omega_{i}=0.9$. Here (a) represents the chaotic state of CTOPLL $\left(k_{m}=0\right)$ and (b) represents the periodic state of DDCPLL $\left(k_{m}=1\right)$ with same second signal.

\section{Conclusion}

We investigate the effects of two co-channel signals on the dynamics of a third order PLL having resonant second order filter through numerical and experimental studies. It is observed that the frequency offset between two signals and their relative amplitudes have pronounced effects on the steady state dynamics of the loop. For a range of values of these parameters a stable PLL enters into chaotic mode through a period doubling route. When the off-set frequency between two input signals is close to the characteristic frequency of the CTOPLL, the susceptibility to chaotic oscillation is more pronounced. We have also proposed a modification in the loop structure and have shown that the modified loop has extended zones of stable operation. The chaotic oscillations of the loop control signal could be suppressed by proper choice of the design parameter of the modified loop. The improved response of the modified third order PLL is due to the inclusion of effective proportionalintegrating-and-derivative control type filter in the loop.

Acknowledgments: Authors acknowledge the infrastructural support provided by the University of Burdwan, India, to carry out the work.

\section{References}

1. F.M. Gardner, Phase lock techniques, $3^{\text {rd }}$ Edition, NJ: John Wiley \& Sons, Hoboken (2005).

2. R.E. Best, Phase-locked loops: design, simulation and applications, McGraw-Hill, New York (2003).
3. P.K. Hanumolu, M. Brownlee, K. Mayaram, and U. K. Moon, Analysis of charge pump phase locked loops, IEEE Trans on Circuits and Systems-I, vol. 51(9), pp. 1665-1673 (2004). 
4. J.R.C. Piqueira, Using bifurcations in the determination of lock in ranges for third-order phase-locked loops, Communications in Nonlinear Science and Numerical Simulation, vol. 14, pp. 23282335 (2009).

5. T. Banerjee and B.C. Sarkar, Chaos and bifurcation in a thirdorder digital phase-locked loop, AEU-International Journal of Electronics and Communications, vol. 62, pp. 86-91 (2008).

6. A. Brambilla, D. Linaro, and M. Storace, Nonlinear behavioral model of charge pump PLLs, International Journal of Circuit Theory and Applications, vol. 41(10), pp. 1027-1046 (2013).

7. B.C. Sarkar, On the joint statistics of amplitude and phase of a signal with co-channel interference, In Proc. of the IEEE, vol. 76(3), pp. 298-299 (1988), DOI:10.1109/5.4414.

8. T. Banerjee and B.C. Sarkar, Phase error dynamics of a class of modified second-order digital phase-locked loops in the background of co-channel interference, Signal Processing, vol. 85(8), pp. 1611-1622 (2005).

9. L.H.A. Monteiro, F.D.N. Favaretto, and J.R.C. Piqueira, Bifurcation analysis for third-order phase-locked loops, IEEE Signal Processing Letter, vol. 11(5), pp. 494-496 (2004).
10. B.A. Harb and M.A. Harb, Chaos and bifurcation in third-order phase-locked loop, Chaos Solitons \& Fractals, vol. 19, pp. 667672 (2004).

11. B.C. Sarkar and S. Chakraborty, Self-oscillations of a third order PLL in periodic and chaotic mode and its tracking in a slave PLL, Communications in Nonlinear Science and Numerical Simulation, vol. 19(3), pp.738-749 (2014).

12. B.C. Sarkar and R. Hati, Chaos from a second order PLL in the presence of CW interference, Electronics Letters, vol. 35(15), pp. 1217-1218 (1999).

13. T. Endo and L. O. Chua, Chaos from phase-locked Loops, IEEE Transactions on Circuits and Systems, vol. 35, pp. 987-1003 (1988).

14. A. Wolf, J.B. Swift, H.L. Swinney, and J.A. Vastano, Determining Lyapunov exponents from a time series, Physica D, vol. 16, pp. 285-317 (1985). 